\title{
ERRATUM
}

\section{Erratum to: Epidemiology of Pneumococcal Disease in a National Cohort of Older Adults}

Haley J. Morrill • Aisling R. Caffrey •

Eunsun Noh $\cdot$ Kerry L. LaPlante

To view enhanced content go to www.infectiousdiseases-open.com

Received: April 17, 2014 / Published online: May 16, 2014

(c) The Author(s) 2014. This article is published with open access at Springerlink.com

Erratum to: Infect Dis Ther

DOI 10.1007/s40121-014-0025-y

The authors would like to make the following adjustment to the above mentioned article. In the published Table 1, total inpatient incidence should be placed under the heading "Inpatient incidence" and not "Outpatient incidence". The correction can be seen in the table below.
Open Access. This article is distributed under the terms of the Creative Commons Attribution Noncommercial License which permits any noncommercial use, distribution, and reproduction in any medium, provided the original author(s) and the source are credited.

The online version of the original article can be found under doi:10.1007/s40121-014-0025-y.

H. J. Morrill · A. R. Caffrey · E. Noh · K. L. LaPlante Infectious Diseases Research Program, Veterans Affairs Medical Center, Providence, Rhode Island, USA

H. J. Morrill · A. R. Caffrey · E. Noh ·

K. L. LaPlante $(\square)$

Department of Pharmacy Practice, College of

Pharmacy, University of Rhode Island, Kingston, Rhode Island, USA

e-mail: kerrylaplante@uri.edu

K. L. LaPlante

Division of Infectious Diseases, Warren Alpert

Medical School of Brown University, Providence,

Rhode Island, USA 
Table 1 Annual incidence of pneumococcal disease by healthcare and age group

\begin{tabular}{|c|c|c|c|c|c|c|c|c|}
\hline \multirow[t]{2}{*}{ Year } & \multicolumn{3}{|c|}{ Outpatient incidence $^{a}$} & \multicolumn{5}{|c|}{ Inpatient incidence ${ }^{b}$} \\
\hline & $\overline{\text { Total }^{c}}$ & 50-64 years & $\geq 65$ years & $\overline{\text { Total }^{c}}$ & 50-64 years & $\geq 65$ years & $\begin{array}{l}\text { Serious } \\
\text { disease }^{d}\end{array}$ & $\begin{array}{l}\text { Invasive } \\
\text { disease }^{\mathrm{e}}\end{array}$ \\
\hline 2002 & 5.8 & 2.4 & 3.4 & 262.3 & 105.5 & 156.8 & 235.3 & 78.1 \\
\hline 2003 & 6.0 & 2.5 & 3.4 & 288.5 & 116.2 & 172.2 & 254.8 & 97.0 \\
\hline 2004 & 5.9 & 2.5 & 3.4 & 270.4 & 116.9 & 153.5 & 234.5 & 88.9 \\
\hline 2005 & 6.0 & 2.7 & 3.3 & 280.6 & 124.7 & 155.9 & 240.0 & 88.6 \\
\hline 2006 & 6.0 & 2.7 & 3.3 & 278.1 & 136.1 & 141.9 & 240.6 & 91.0 \\
\hline 2007 & 5.9 & 2.7 & 3.2 & 277.7 & 135.5 & 142.2 & 230.1 & 87.5 \\
\hline 2008 & 5.6 & 2.6 & 3.0 & 309.9 & 147.1 & 162.8 & 264.0 & 97.7 \\
\hline 2009 & 4.9 & 2.2 & 2.7 & 307.4 & 148.7 & 158.7 & 258.5 & 91.6 \\
\hline 2010 & 4.0 & 1.8 & 2.2 & 305.4 & 144.3 & 161.1 & 253.4 & 92.6 \\
\hline 2011 & 2.9 & 1.3 & 1.6 & 328.1 & 154.5 & 173.5 & 264.7 & 94.6 \\
\hline $\begin{array}{l}\text { Annualized percent } \\
\text { change (\%) }\end{array}$ & -3.5 & -4.1 & -3.1 & 0.2 & -0.6 & 0.7 & 0.1 & -1.0 \\
\hline$P$ value & $<0.001$ & 0.001 & 0.003 & 0.846 & 0.391 & 0.533 & 0.888 & 0.454 \\
\hline
\end{tabular}

Incidence based on all positive Streptococcus pneumoniae cultures from any site, unless otherwise indicated

a Number of infections per 100,000 clinic visits

b Number of infections per 100,000 hospital visits

c Includes all patients aged $\geq 50$ years

$\mathrm{d}$ Includes only serious pneumococcal infections (pneumonia, bacteremia, and meningitis)

e Includes only invasive pneumococcal disease (bacteremia, meningitis, and bacteremic pneumonia) 